\title{
Reposition of Cardiac Implantable Electronic Devices Using Pectoralis Major Muscle Pocket
}

\author{
Hyun Sik Yoon, Young Cheon Na \\ Department of Plastic and Reconstructive Surgery, Wonkwang University Hospital, Iksan, Korea
}

\begin{abstract}
Increasing implantations of cardiac implantable electronic devices mean that complications such as infections and device exposures have also surged. A 66-year-old man visited our clinic with recurring cardiac device exposure due to skin defects. He had undergone pacemaker insertion by a cardiologist 7 years earlier, and after experiencing device exposure, had had two revision operations, 1 year ago and then again 3 months prior. The patient's pacemaker function test results and laboratory findings were normal and the clinical signs convinced us that his condition was a non-infectious reaction. We therefore repositioned the device to a different plane. With minimal dissection, we created in the shape of a reverse trapezoid a specific pocket slightly smaller than the device to reposition the device under the pectoralis major muscle. The patient recovered without complications. Though principle dictates that an exposed device should be removed and re-implanted in a new site, conservative treatment is attempted in some cases because of the high cost, potential for complications during removal, and specific circumstances of the patient. Repositioning the device to change the plane using a specific pectoralis major pocket can serve as an option for certain cases.
\end{abstract}

Keywords: Defibrillators, implantable; Pacemaker; Postoperative complications

\section{Introduction}

Cardiac implantable electronic devices (CIEDs) can be broadly divided into permanent pacemakers (PPMs), implantable cardioverter-defibrillators (ICDs) and cardiac resynchronization therapy (CRT) devices. A PPM detects an irregular heartbeat (arrhythmia) and regulates the heartbeat, while an ICD is the treatment of choice for structural heart disease and symptomatic sustained ventricular tachycardia or ventricular fibrillation and also a prophylactic therapy for sudden cardiac death [1,2]. CRT devices, meanwhile, are used to improve the heart function and rate of survival, preventing sudden death in patients who show progressive heart failure despite adequate medication [3,4]. The increase in cases of cardiac diseases requiring CIED treatments has led to higher rates of device implantation, hence the increase of complications of CIEDs [5]. CIEDs are usually placed subcutaneously, which in elderly patients may bring about complications such as device exposure due to skin defects and device twiddling [6]. CIED-related complications remain a serious problem with increased morbidity and mortality rates and healthcare costs because the cardiac devices that control life-threatening symptoms are expensive and require an invasive procedure for implantation [5]. In principle, reimplantation is required after the device is removed due to infection; however, in cases of non-infectious device exposure, conservative treatments such as device repositioning are possible [5,7-9]. Here we report the successful use of a pectoralis major pocket for device repositioning in a case of recurrent exposure of a permanent pacemaker. The study was performed in accordance

\section{(1) \\ Case Report \\ Received: August 23, 2019 \\ Revised: September 9, 2019 \\ Accepted: September 10, 2019 \\ Corresponding author: \\ Young Cheon Na, M.D., Ph.D. \\ Department of Plastic and Reconstructive Surgery, Wonkwang University Hospital, 895 Muwang-ro, Iksan 54538, Korea \\ Tel: +82-63-859-1570 \\ Fax: +82-63-858-3922 \\ E-mail: ycnadr@hanmail.net}

This is an Open Access article distributed under the terms of the Creative Commons Attribution Non-Commercial License (http://creativecommons.org/licenses/by-nc/4.0/) which permits unrestricted non-commercial use, distribution, and reproduction in any medium, provided the original work is properly cited.

(c) 2019 Korean Wound Management Society 
with the principles of the Declaration of Helsinki. Written informed consent was obtained.

\section{Case}

A 66-year-old man visited our clinic with recurring device exposure due to a skin defect where his pacemaker had been inserted. The patient had undergone pacemaker insertion by a cardiologist 7 years prior. Afterwards, the patient's device had become exposed and he had two revision operations at a local hospital 1 year before and then 3 months prior to coming to our clinic. On preoperative evaluation, the device was exposed

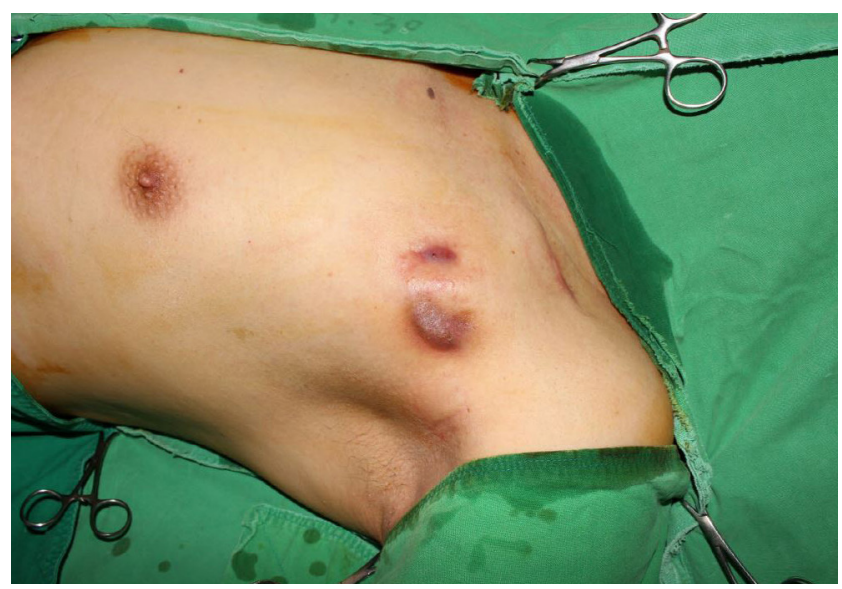

Fig. 1. Pre-operation photograph. On preoperative evaluation, the device was exposed with a $0.5 \mathrm{~cm} \times 1.0 \mathrm{~cm}$ skin defect, and the surrounding skin was thin.

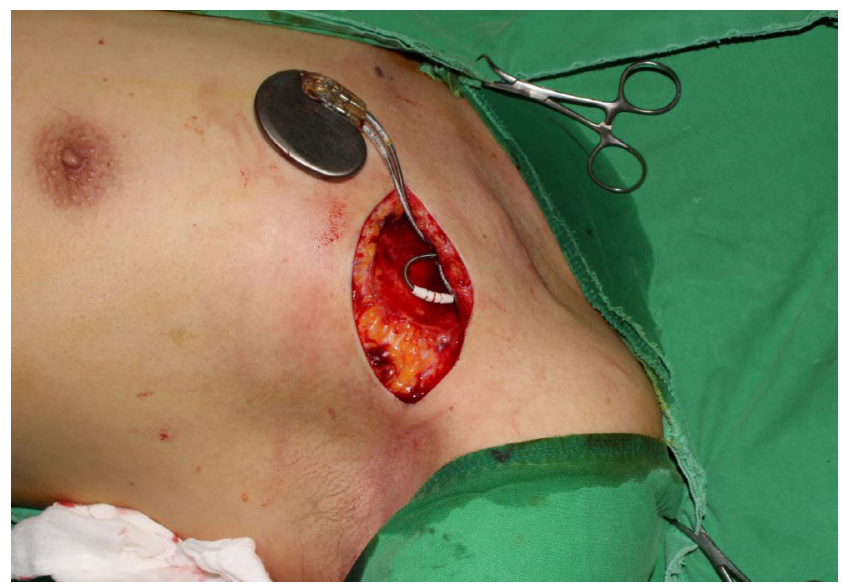

Fig. 2. Intra-operation finding. During the operation, we performed debridement of unhealthy granulation and were able to confirm that there was no lead infection. with a $0.5 \mathrm{~cm} \times 1.0 \mathrm{~cm}$ skin defect and the surrounding skin had thinned (Fig. 1). However, a test of the pacemaker functions found it to be normal; there were no systemic symptoms or localized inflammatory signs, the laboratory findings were also normal, and neither was any growth seen on the wound culture. Based on the patient's clinical symptoms and signs, his condition was considered a non-infectious reaction. Therefore, we repositioned the device instead of removing it, changing its plane from subcutaneous to submuscular. With the patient under general anesthesia, we debrided the unhealthy granulation and confirmed there was no lead infection (Fig. 2). We created a pocket under the pectoralis major muscle to

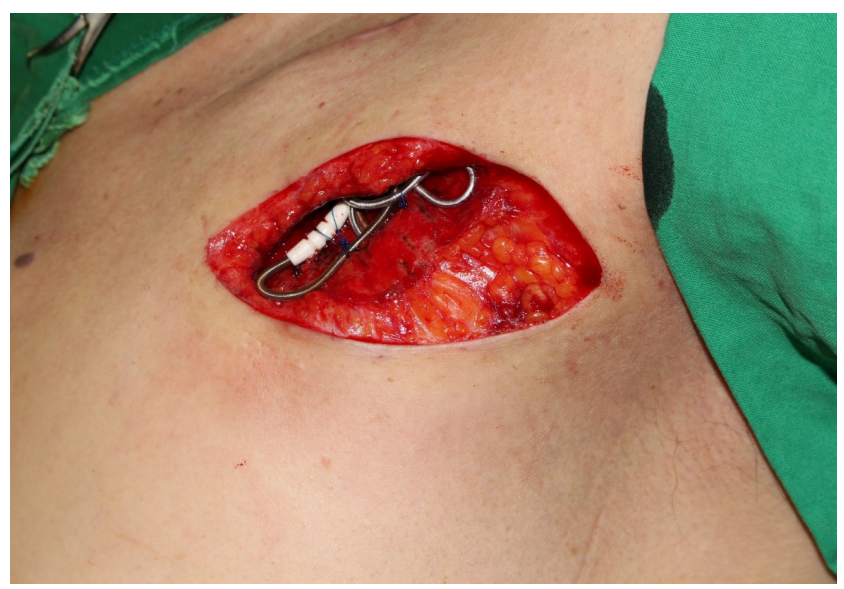

Fig. 3. Operation techniques. The pocket was made with finger dissection after minimal incision, was slightly smaller than a pacemaker, and in the shape of a reverse trapezoid like the device itself. The device was inserted with a gentle push and fixed firmly with multiple non-absorbable anchoring sutures to minimize the device migration.

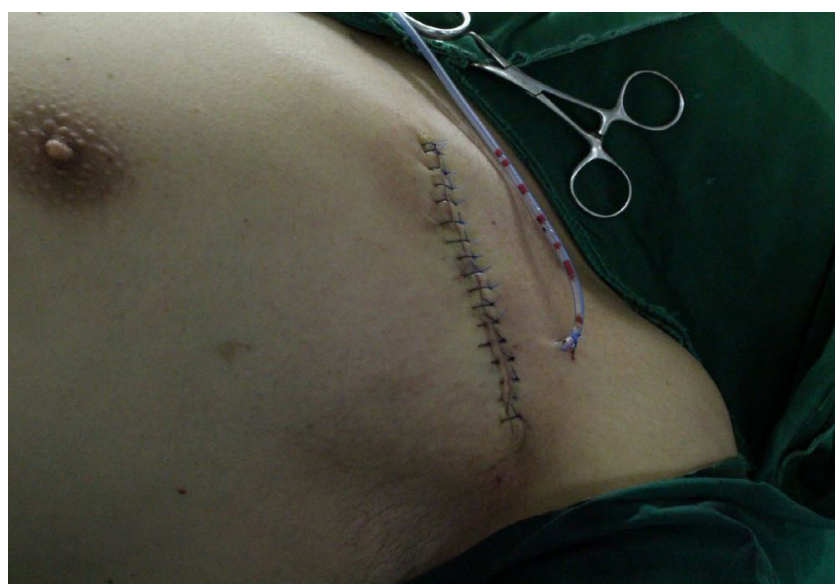

Fig. 4. Post-operation photograph. Fasciocutaneous advancement flap is performed. 


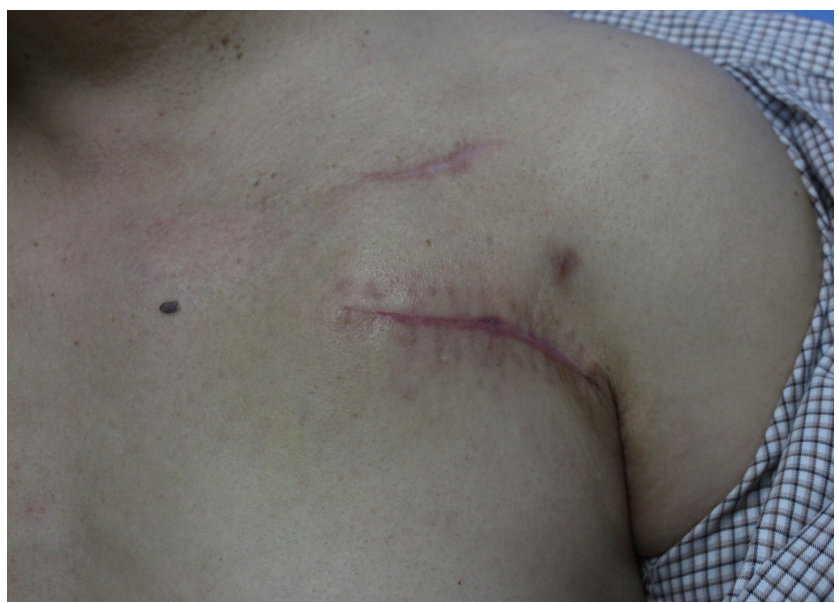

Fig. 5. Photograph at the 2 months follow-up visit. The patient had recovered without complications, and later received scar management.

position the device. The pocket, in the shape of a reverse trapezoid and slightly smaller than the device, was made with finger dissection after minimal incision. The device was inserted with a gentle push and fixed firmly with multiple non-absorbable anchoring sutures using \#5-0 Prolene to minimize its migration (Fig. 3). The defect was covered with a local advancement flap at the fasciocutaneous level in both lateral margins (Fig. 4). The patient recovered without complications and received scar management (Fig. 5).

\section{Discussion}

Technological advancement has reduced the size of cardiac devices, making it possible to implant them in the pectoral position, simplify the implantation procedure by avoiding the need for tunneling to the abdomen, and use them in pediatric patients. Defibrillation efficacy was also enhanced $[2,10]$. Pockets for the device can now be created in the axilla, abdomen, and pectoral regions. Though implanting a cardiac device in the pectoral position is now a routine procedure, physicians continue to strive to find a more efficient way with fewer complications and better cosmetic results [11].

Devices in pectoral regions are either placed in subcutaneous or submuscular positions, depending on the plane. The subcutaneous position makes it easy to create pockets, causes less pain, facilitates replacement of the generator, leads to less neurovascular injury, and is cost effective because it can be performed under local anesthesia. However, it also carries the risks of skin ulceration, device exposure, device twiddling, poor cosmetic contour, and device damage when subject to external impact. This position also carries the possibility of infection and exposure because of the weight and size of the device. The devices have the tendency to migrate downwards due to gravity and respiratory movements, and the tissue covering the device is continuously damaged by friction, pressure, and shear force, especially in elderly individuals with lax skin [7]. The idea of positioning the device submuscularly therefore emerged after a study that aimed to solve the frequent wound complications arising in the subcutaneous position. The submuscular position is to place the device in an intramuscular pocket between the pectoralis major and minor or in a submuscular pocket in the pectoralis muscles. The adipose tissue affords protection, rendering the device more resistant to external impact. The submuscular position also brings the advantages of better cosmetic results and lower risk of migration. However, the difficulty of creating a pocket, the severe pain, and the need for conscious sedation are some drawbacks, and for patients taking anticoagulants, the insertion of a drain is also recommended to prevent hematoma $[6,10,11]$. According to Gold et al. [10], there was no significant difference in complications between the subcutaneous and submuscular positions. Therefore, the subcutaneous position, which is simpler, less painful, and cost effective and can be performed under local anesthesia, is currently performed as the default procedure.

CIED-related complications can be grouped by their causes into procedure-related complications (refractory heart failure, stroke, pneumothorax, cardiac tamponade, and coronary sinus perforation), lead-related complications (lead displacement, lead fracture, and insulation defect), and pocket-related complications (skin erosion, hematoma, seroma, wound infection, and device migration) [1]. The most common is a generator pocket infection followed by device-related endocarditis. Despite improvements in implantation techniques, infections remain a serious problem with increased morbidity and mortality rates and financial costs [5]. The clinical presentation of patients with infections is as follows: (1) systemic symptoms (fever, chills, malaise, anorexia, nausea, etc.); (2) localized inflammatory signs at the generator site (erythema, pain, swelling, warmth, tenderness, drainage, skin ulceration, lead erosion, etc.); and (3) laboratory abnormalities (leukocytosis, anemia, high erythrocyte sedimentation rate, positive blood culture, etc.) $[5,8]$. In principle, an infection after implantation of a cardiac device requires complete removal of the device, followed by antibiotic therapy for at least for 2 weeks and reimplantation in a new site [8]. In the case of lead infections, device removal is essential because of the risk of endocarditis 
and sepsis [5].

However, surgeons encounter the dilemma of whether or not to remove the cardiac device in cases of simple device exposure without infection. Notwithstanding the possibility of fatal complications such as myocardial perforation, valve tearing, and venous laceration during lead removal and specific conditions such as venous occlusion or an axillary jugular arteriovenous shunt, many surgeons attempt conservative treatment because of the high costs of the devices. Conservative treatment refers to simple revision or repositioning to another plane after appropriate debridement. Determining whether an implantable device is infectious or not is yet another challenge, even if the culture result was negative, as it is usually impossible to distinguish between a non-infectious reaction and a low-grade infection [7]. As discussed above, a thorough analysis of the patient's systemic symptoms, local signs of inflammation and laboratory abnormalities is required to determine whether the implantable devices are infected [12]. We considered this case a non-infectious reaction based on the patient's clinical symptoms, signs and laboratory findings.

There is currently no definite guideline for managing CIEDrelated complications. Therefore, the decision regarding device removal should not be automatic and must be made on a caseby-case basis for each patient [9]. We believe that using a specific pectoralis major pocket to change the plane of the device not only has the advantages of submuscular implantation such as better cosmetic results and protection against external impacts, but is also easier to perform than a traditional submuscular implantation because it requires muscle dissection only to the extent that the device is inserted with a gentle push. The use of anchoring sutures can minimize migration that causes friction pressure and shear forces, reducing recurrence of device exposure and increasing the likelihood of success. Therefore, in cases of non-infectious device exposure, repositioning using a specific pocket to change the plane of the device from subcutaneous to submuscular is a valuable option.

\section{Conflict of interest}

No potential conflicts of interest relevant to this article are reported.

\section{Acknowledgments}

Hyun Sik Yoon https://orcid.org/0000-0002-2294-5465

Young Cheon Nahttps://orcid.org/0000-0003-3136-0351

\section{References}

1. Alter P, Waldhans S, Plachta E, et al. Complications of implantable cardioverter defibrillator therapy in 440 consecutive patients. Pacing Clin Electrophysiol 2005;28:926-32.

2. Oh JH, Kim CM, Song SY, et al. Transaxillary subpectoral placement of cardiac implantable electronic devices in young female patients. Arch Plast Surg 2017;44:34-41.

3. Bradley DJ, Bradley EA, Baughman KL, et al. Cardiac resynchronization and death from progressive heart failure: a metaanalysis of randomized controlled trials. JAMA 2003;289:73040.

4. Park HS. Implantable cardioverter-defibrillator and cardiac resynchronization therapy. Korean J Med 2016;90:210-6.

5. Sohail MR, Uslan DZ, Khan AH, et al. Management and outcome of permanent pacemaker and implantable cardioverterdefibrillator infections. J Am Coll Cardiol 2007;49:1851-9.

6. Relland JY. Permanent pacemaker implantation: retropectoral position and lead insertion through the subclavian vein. Ann Thorac Surg 1990;49:835-6.

7. Chari PS, Suri RK. Skin cover for exposed cardiac pacemakers. Br J Plast Surg 1977;30:231-4.

8. Chua JD, Wilkoff BL, Lee I, et al. Diagnosis and management of infections involving implantable electrophysiologic cardiac devices. Ann Intern Med 2000;133:604-8.

9. Tarakji KG, Chan EJ, Cantillon DJ, et al. Cardiac implantable electronic device infections: presentation, management, and patient outcomes. Heart Rhythm 2010;7:1043-7.

10. Gold MR, Peters RW, Johnson JW, et al. Complications associated with pectoral cardioverter-defibrillator implantation: comparison of subcutaneous and submuscular approaches. Worldwide Jewel Investigators. J Am Coll Cardiol 1996;28: 1278-82.

11. Rajappan K. Permanent pacemaker implantation technique: part I: arrhythmias. Heart 2009;95:259-64.

12. Koutentakis M, Siminelakis S, Korantzopoulos P, et al. Surgical management of cardiac implantable electronic device infections. J Thorac Dis 2014;6 Suppl 1:S173-9. 